\title{
Corresponding Articular Cartilage Thickness Measurements in the Knee Joint by Modelling the Underlying Bone
}

\author{
Tomos G. Williams ${ }^{1}$, Christopher J. Taylor ${ }^{1}$, ZaiXiang Gao ${ }^{1}$, and \\ John C. Waterton ${ }^{2}$ \\ 1 Imaging Science and Biomedical Engineering, University of Manchester, \\ Manchester, U.K. \\ 2 Enabling Science \& Technology, AstraZeneca, Alderley Park, Macclesfield, \\ Cheshire, U.K.
}

\begin{abstract}
We present a method for corresponding and combining cartilage thickness readings from a population of patients using the underlying bone structure as a reference. Knee joint femoral bone and cartilage surfaces are constructed from a set of parallel slice segmentations of MR scans. Correspondence points across a population of bone surfaces are defined and refined by minimising an objective function based on the Minimum Description Length of the resulting statistical shape model. The optimised bone model defines a set of corresponding locations from which 3D measurements of the cartilage thickness can be taken and combined for a population of patients. Results are presented for a small group of patients demonstrating the feasibility and potential of the approach as a means of detecting sub-millimetre cartilage thickness changes due to disease progression.
\end{abstract}

\section{Introduction}

Osteoarthritis is a major cause of suffering and disability. This has lead to a growing demand for effective alternatives to surgical treatments, which are only suitable in extreme cases [2]. Osteoarthritis causes degeneration of articular cartilage, although characterising cartilage and bone changes during disease progression is still the subject of current research [12. MR imagery of the knee can be used to monitor cartilage damage in vivo 315. Most studies suggest that total cartilage volume and mean thickness are relatively insensitive to disease progression 24 4,17] though there are some conflicting results 2014]. There is evidence to suggest that osteoarthritis causes regional changes in cartilage structure with some regions exhibiting thinning or loss of cartilage whilst swelling may occur elsewhere on the articular surface. For this reason, localised measures of cartilage thickness are likely to provide a fuller picture of the changes in cartilage during the disease process.

In healthy subjects knee articular cartilage is, on average, only $2 \mathrm{~mm}$ thick [5]8] and thickness changes over the short time scale useful in drug development 
(6-12 months), are likely to be in the sub-millimetre region. It is unlikely that such small changes will be detected in individual pairs of MR scans given practical scan resolutions and segmentation accuracies. Previous work has shown that small but systematic changes in thickness between two time points can be measured in a group of subjects by registering the set of cartilage segmentations and computing mean change at each point of the cartilage surface 19. These studies used elastic registration of the segmented cartilage shapes in normal volunteers. This has two obvious problems: there is no guarantee that anatomically equivalent regions of cartilage are corresponded, even in normal subjects, and the correspondences become unpredictable when the cartilage shape changes during disease (particularly when there is loss from the margins).

In this paper we propose using the underlying bone as an anatomical frame of reference for corresponding cartilage thickness maps between subjects over time. This has the advantage that anatomically meaningful correspondences can be established, that are stable over time because the disease does not cause significant changes in overall bone shape. We find correspondences between anatomically equivalent points on the bone surface for different subjects using the minimum description length method of Davies el al. [6] which finds the set of dense correspondences between a group of surfaces that most simply account for the observed variability. This allows normals to be fired from equivalent points on each bone surface, leading to directly comparable maps of cartilage thickness.

\section{Method}

\subsection{Overview}

MR images of the knee were obtained using T1 weighted fat-suppressed spoiled $3 \mathrm{D}$ gradient echo sequence to visualise cartilage and a T2 weighted sequence to visualise the endosteal bone surface, both with $0.625 \times 0.615 \times 1.6 \mathrm{~mm}$ resolution. Semi-automatic segmentations of the femoral cartilage and endosteal surface of the femur were performed slice-by-slice using the EndPoint software package (Imorphics, Manchester, UK). These slice segmentations were used to build continuous 3D surfaces, an MDL model of the bone was constructed and standardised thickness maps were generated as described in some detail below. The data used contained images of both left and right knees. To simplify subsequent processing, all left knees were reflected about the medial axis of the femur so they could be treated as equivalent to right knees.

\subsection{Surface Generation}

To provide a common reference across all examples, each bone segmentation was truncated to include a length of femoral shaft proportional to the width of the femoral head. Where adjacent segmentations differed significantly, additional contour lines were inserted at the mid line of the two segmentations.

Surface construction from the cartilage segmentations proved more challenging due to significant variation between neighbouring slices and the thin, curved 
shape of the cartilage. Various documented approaches such as NUAGES triangulation [10] and Shape Based Interpolation [16] proved unable to produce plausible surfaces so an alternative surface construction method specifically for articular cartilage was developed.

Post processing of the segmentations was needed to identify the exosteal surface or bone-cartilage interface and outer surface of the cartilage. This simplified surface construction by allowing the structure connecting each segment to be determined by the inner surface and then inherited by the outer surface. The segments' connection sequence was also specified. Both the inner/outer surface and segment connection sequence operations were performed automatically with manual correction if required.

During cartilage surface constriction, regions of the segments were categorised as either spans (connecting two segments) or ridges (overhangs where the surface is closed and connected to itself). The underlying structures were represented as quadrilateral meshes and connected to ensure that the surface was closed. Surface generation was performed by triangulation of this mesh.

\subsection{Bone Statistical Shape Model}

We adopted the method of Davies et al. 6 to find an optimal set of dense correspondences between the bone surfaces The bone surfaces were pre-processed to move their centroids to the origin and scaled so that the Root Mean Square of the vertices' distance from the centroid was unity. This initial scaling facilitated model optimisation by minimising the effect of differences in the overall size of the examples on the shape model. Additional pose refinement is incorporated in the optimisation process.

Each bone surface was mapped onto a common reference; an unit sphere is chosen since it possessed the same topology as the bone and provides a good basis for the manipulation of the points by reducing the number of point parameters from the three Cartesian points of the shape vertices to two spherical coordinates. The diffusion method of Brechbühler [1] was used to produce the spherical mappings. A set of equally spaced points were defined on the surface of the unit sphere and mapped back onto each bone surface by finding their position on the spherically mapped surfaces - the triangle on which they are incident and their precise position on this triangle in barycentric coordinates and computing the same location on the corresponding triangle on the original surface. This provided a first approximation to a set of corresponding points across the population of bone surfaces. At this stage there is, however, no reason to expect anatomical equivalence between corresponding points

The automatic model optimisation method of Davies at al. 7] is based on finding the set of dense correspondences over a set of shapes that produce the 'simplest' linear statistical shape model. A minimum description length (MDL) objective function is used to measure model complexity [6], and optimised numerically with respect to the correspondences. The basic idea is that 'natural' correspondences give rise to simple explanations of the variability in the data. 
One shape example was chosen as a reference shape and the positions of its correspondence points remained fixed throughout. The optimisation process involved perturbing the locations of the correspondence points of each shape in turn optimising the MDL objective function. Two independent methods of modifying the positions of the correspondence points were used: global pose and local Cauchy transform perturbations on the unit sphere. Global pose optimisation involved finding the six parameters ( $\mathrm{x}$ y z translation and rotation) applied to the correspondence points of a shape that minimise the objective function. Reducing the sizes of the shapes trivially reduces the MDL objective function so the scale of each shape was fixed throughout the optimisation.

Local perturbation of the correspondence points on the unit sphere, guaranteed to maintain shape integrity, is achieved by using Cauchy kernels to locally re-parametrise the surface. Each kernel has the effect of attracting points toward the point of application. The range of the effect depends on the size of the kernel. One step in the optimisation involved choosing a shape at random, optimising the objective function with respect to the pose, place a kernel of random width (from an interval) at random points on the unit sphere and finding the amplitude (size of effect) that optimised the objective function. This was repeated until convergence.

\subsection{Measuring Cartilage Thickness from the Bone}

Different measures of cartilage thickness have been proposed, all taking their initial reference points from the exosteal surface of the cartilage [5,11,13,18]. Our work differs in that the reference points for the measurements are taken from the endosteal surface of the cortical bone along 3D normals to the bone surface at the correspondence points determined as described above. On firing a normal out of the bone surface, the expected occurrence is to either find no cartilage, as is the case around regions of the bone not covered by any articular cartilage, or intersect with the cartilage surface at two points, on its inner and outer surfaces. The thickness of the cartilage is recorded as the distance along the bone normal between its points of intersection with the inner and outer cartilage surface. By taking a cartilage thickness reading at each correspondence point a cartilage thickness map can be drawn onto the bone surface. Sets of cartilage thickness readings taken at the corresponding points, defined by the MDL model, can be combined for sets of patients and compared between different time-points.

\section{Results}

18 sets of bone segmentations for 6 at risk patients were processed. The data was equally divided between two time-points ( 0 and 6 months). With this small set of data the intention was to demonstrate the feasibility of the approach rather than deduce any characteristics of cartilage thickness change during arthritic disease progression. Surface construction from the bone segmentations yielded on average 4168 (range 3154-4989) vertices and 8332 (6304-9974) triangles. 4098 


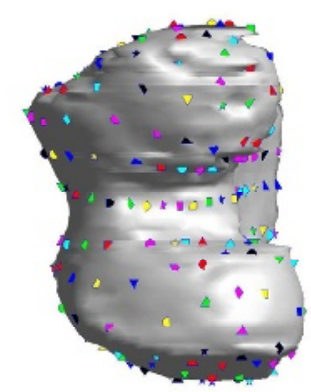

(a) $\mathrm{AB}$

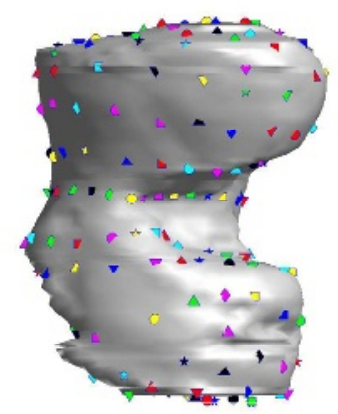

(c) ES

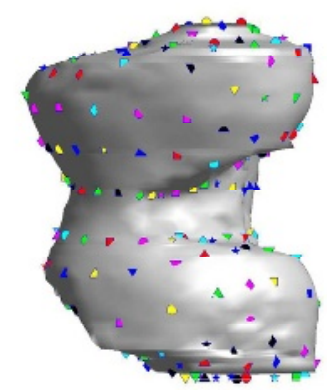

(b) DA

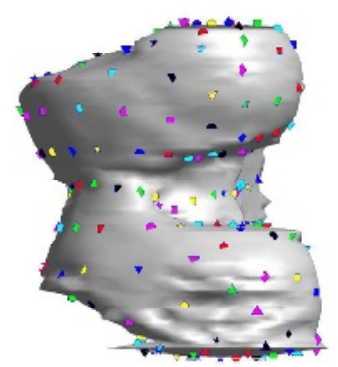

(d) $\mathrm{IH}$

Fig. 1. A sub-set of the correspondence points shown on 4 of the population of bone surfaces. The objective is for the corresponding points to reside on the same anatomical regions of the bone across all the shapes. These plots illustrate that the model has been able to provide good correspondence across the population of shapes.

correspondence points were defined on the unit sphere and projected onto each bone surface, from which the statistical model was built and refined. Figure 1 shows a proportion of the resultant correspondence points projected onto a subset of the population. It can be seen that the correspondences are anatomically plausible.

Only a proportion of the bone correspondence points reside on regions of the surface which are covered by cartilage. Typically, 950 of the 4098 corresponding measurement points resulted in cartilage thickness readings. For a cartilage endosteal surface area of $4727 \mathrm{~mm}^{2}$ this represents coverage of 0.201 thickness 

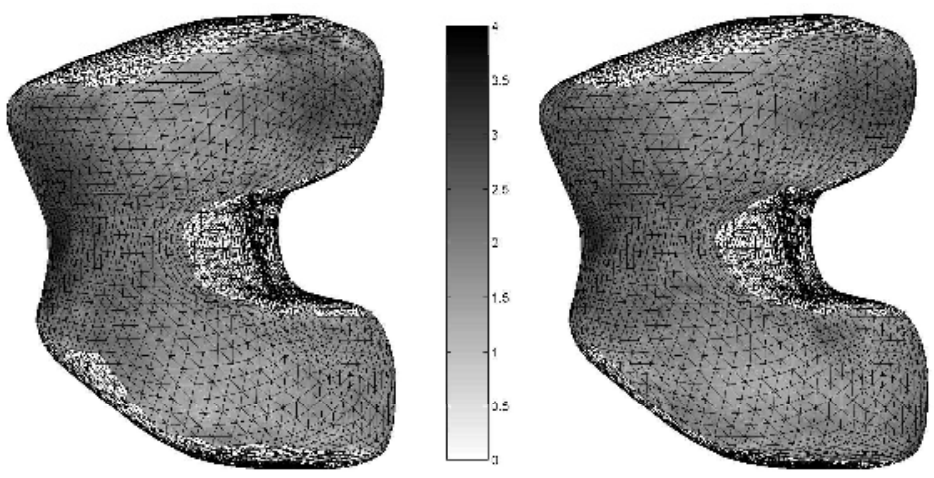

(a) TP1 and TP2
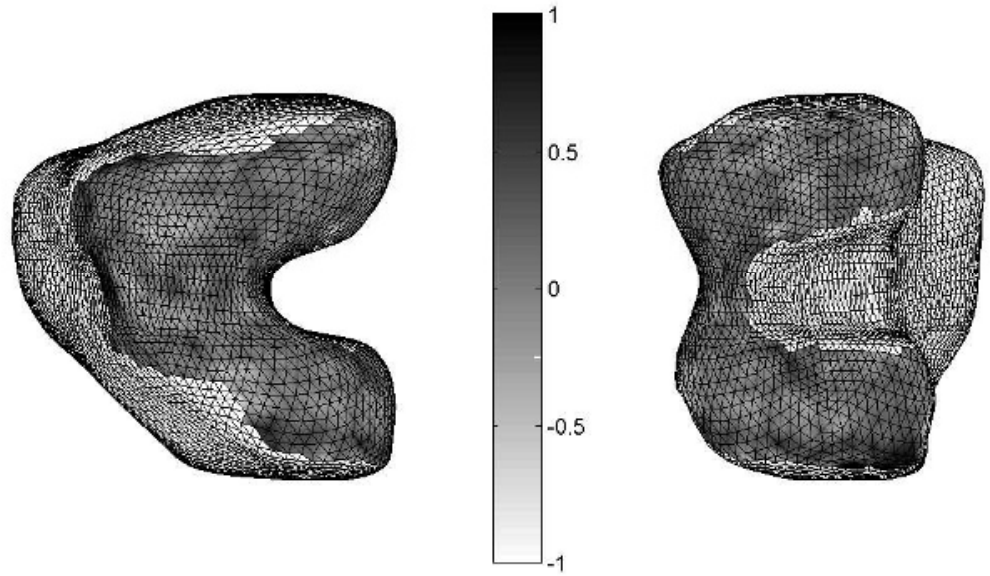

(b) TP2-TP1 viewed from two different angles

Fig. 2. Mean cartilage thickness from the time-point 1 and time-point $2(0$ and 6 months) segmentations and the difference all represented as cartilage thickness mapped onto the average bone shape. Regions where swelling of the cartilage occurs are coloured dark while white indicates thinning.

readings per $\mathrm{mm}^{2}$ and an average separation of $2.23 \mathrm{~mm}$ between readings; sufficient coverage and number of points to perform statistical analysis of the data.

Figure 2 illustrates how populations of results can be combined and compared. Mean thickness measurements for each corresponding point are displayed as colour maps on the mean bone shape. The results for time points 0 and 6 months scans are illustrated together with the difference between these aggregate 
maps. The difference map demonstrates thinning of cartilage in the load-bearing regions such as the patellofemoral (middle left) and medial tibiofemoral (upper right) compartments which is analogous to the finding reported in a diurnal study [19]. A larger study will be required to draw firm conclusions.

\section{Conclusions and Further Work}

We have demonstrated the feasibility of using the underlying bone as a reference for cartilage thickness measurements. The bone provides a stable reference for examining surfaces built from segmentations of cartilage scans taken at different time points. Inter-patient comparisons can be achieved by building and optimising a Statistical Shape Model of the femoral head. Cartilage thickness measurements are taken over all bone examples at the resultant corresponding locations which allows for the aggregation of results from a population of patients and comparisons between sets of patients.

The approach was illustrated by applying it to a small population of 18 bone segmentations divided between two time-points. Two sets of measurements were combined to produce mean thickness maps which were then compared to each other to illustrate a comparative cartilage thickness map illustrating regional cartilage thickness changes.

The immediate requirement is to complete larger scale experiments and extend the approach to the other (tibial and patellal) articular surfaces of the knee joint. Further refinement of the surface construction and image registration of the bone and cartilage scans could yield greater accuracy in cartilage thickness measurements.

\section{References}

1. C. Brechbühler, G. Gerig, and O. Kubler. Parametrization of closed surfaces for 3-D shape-description. Computer Vision and Image Understanding, 61(2):154-170, 1995.

2. J. A. Buckwalter, W. D. Stanish, R. N. Rosier, R. C. Schenck, D. A. Dennis, and R. D. Coutts. The increasing need for nonoperative treatment of patients with osteoarthritis. Clin. Orthop. Rel. Res., pages 36-45, 2001.

3. R. Burgkart, C. Glaser, A. Hyhlik-Durr, K. H. Englmeier, M. Reiser, and F. Eckstein. Magnetic resonance imaging-based assessment of cartilage loss in severe osteoarthritis - accuracy, precision, and diagnostic value. Arthritis Rheum., 44:2072-2077, 2001.

4. F. M. Cicuttini, A. E. Wluka, and S. L. Stuckey. Tibial and femoral cartilage changes in knee osteoarthritis. Ann. Rheum. Dis., 60:977-980, 2001.

5. Z. A. Cohen, D. M. McCarthy, S. D. Kwak, P. Legrand, F. Fogarasi, E. J. Ciaccio, and G. A. Ateshian. Knee cartilage topography, thickness, and contact areas from MRI: in-vitro calibration and in-vivo measurements. Osteoarthritis and Cartilage, 7:95-109, 1999.

6. Rhodri H Davies, Carole J Twining, Tim F Cootes, John C Waterton, and Chris T Taylor. A minimum description length approach to statistical shape modelling. IEEE Trans. on Medical Imaging, 21(5):525-537, May 2002. 
7. Rhodri H Davies, Carole J Twining, Tim F Cootes, John C Waterton, and Chris T Taylor. 3D statistical shape models using direct optimisation of description length. In rth European Conference on Computer Vision, pages 3-21, 2002.

8. F. Eckstein, M. Winzheimer, J. Hohe, K. H. Englmeier, and M. Reiser. Interindividual variability and correlation among morphological parameters of knee joint cartilage plates: analysis with threedimensional MR imaging. Osteoarthritis Cartilage, 9:101-111, 2001.

9. Stephen J Gandy, Alan D Brett, Paul A Dieppe, Michael J Keen, Rose A Maciwicz, Chris J Taylor, and John C Waterton. No change in volume over three years in knee osteoarthritis. In Proc. Intl. Soc. Magnetic Resonance, page 79, 2001.

10. Bernhard Geiger. Three-dimensional modeling of human organs and its application to diagnosis and surgical planning. Thèse de doctorat en sciences, École Nationale Supérieure des Mines de Paris, France, 1993.

11. J Hohe, G Ateshian, M Reiser, KH Englmeier, and F Eckstein. Surface size, curvature analysis, and assessment of knee joint incongruity with MRI in-vivo. Magnetic Resonance in Medicine, 47(3):554-561, 2002.

12. J. A. Martin and J. A. Buckwalter. Aging, articular cartilage chondrocyte senescence and osteoarthritis. Biogerontology, 3:257-264, 2002.

13. C. A. McGibbon, D. E. Dupuy, W. E. Palmer, and D. E. Krebs. Cartilage and subchondral bone thickness distribution with MR imaging. Acad. Radiol., 5:20-25, 1998.

14. C. G. Peterfy, C. F. Vandijke, D. L. Janzen, C. C. Gluer, R. Namba, S. Majumdar, P. Lang, and H. K. Genant. Quantification of articular-cartilage in the knee with pulsed saturation-transfer subtraction and fat-suppressed MR-imaging - optimization and validation. Radiology, 192:485-491, 1994.

15. Charles G Peterfy. Magnetic resonance imaging in rheumatoid arthritis: Current status and future directions. Journal of Rheumatology, 28(5):1134-1142, May 2001.

16. S. P. Raya and J. K. Udupa. Shape-based interpolation of multidimensional objects. IEEE Trans. on Medical Imaging, 9(1):32-42, 1990.

17. T. Stammberger, J. Hohe, K. H. Englmeier, M. Reiser, and F. Eckstein. Elastic registration of $3 \mathrm{D}$ cartilage surfaces from MR image data for detecting local changes in cartilage thickness. Magn. Reson. Med., 44(4):592-601, 2000.

18. S. K. Warfield, M. Kaus, F. A. Jolesz, and R. Kikinis. Adaptive, template moderated, spatially varying statistical classification. Med. Image Anal., 4(1):43-55, 2000 .

19. John C Waterton, Stuart Solloway, John E Foster, Michael C Keen, Stephen Grady, Brian J Middleton, Rose A Maciewicz, Iain Watt, Paul A Dieppe, and Chris J Taylor. Diurnal variation in the femoral articular cartilage of the knee in young adult humans. Magnetic Resonance in Medicine, 43:126-132, 2000.

20. A. E. Wluka, S. Stuckey, J. Snaddon, and F. M. Cicuttini. The determinants of change in tibial cartilage volume in osteoarthritic knees. Arthritis Rheum., 46(8):2065-2072, August 2002. 